\title{
A MODEL FOR CONTRAST DISCRIMINATION WITH INCREMENTAL AND DECREMENTAL TEST PATCHES
}

\author{
Fred Kingdom' and Bernard Moulden² \\ 'Physiological Laboratory, Downing Street, Cambridge CB2 3EG, U.K. and 'Department of Psychology. \\ University of Western Australia. Nedlands, Perth, WA 6009. Australia
}

(Receited 29 September 1989; in retised form 3 May 1990)

\begin{abstract}
Whittle [Vision Research, 26, 1677 (1986)] has shown that the metric of contrast $W=\Delta L / L_{\operatorname{mix}}$ ( $\Delta L=$ difference in luminance between test patch and background, $L_{\min }=$ the smaller of the luminance of the background or test patch) is able to provide a unifying description of the pattern of contrast discrimination thresholds for pairs of test patches set against a common background. In particular the metric $W$ unifies the pattern of discrimination thresholds for both increment and decrement pairs. We argue that while $W$ provides a good mathematical description of Whittle's data it is functionally implausible since it implies that the component of the stimulus which sets the adaptational level for increments is different from that which sets the adaptational level for decrements. We argue that the metric $G=\ln \left(L / L_{b}\right)\left(L=\right.$ test patch luminance, $L_{b}=$ background luminance) is physiologically more plausible than $W$ and show that $G$ can provide at least as good a fit as $W$ to Whittle's data when incorporated in a transfer function of the form $R_{G}=k G^{-n}$, with $n$ set to 0.69 . The fit to the data can be improved still further if a parameter representing the non-linearity in the gain-luminance function at low luminances is included in the $R_{G}$ equation. The theoretical implications for retinal gain mechanisms are discussed.
\end{abstract}

Contrast discrimination thresholds Inerements Decrements

\section{INTRODUCTION}

In an excellent series of experiments (summarised in Whittle, 1986) Whittle and his coworkers described the ability of human observers to discriminate between pairs of luminance increments (Whittle \& Swanston, 1974) and luminance decrements (Whittle 1986) over a wide range of physical contrasts (more than 6 $\log$ units) and background luminances ( $3 \mathrm{log}$ units). In this paper we aim to provide a model to account for Whittle's (1986) data.

We begin by describing the broad features of Whittle's findings. A summary of the terminology employed by Whittle and ourselves is diagrammatically illustrated in Fig. 1. Full details of Whittle's procedures are given in Whittle (1986). At very low values of $\Delta L$ (see Fig. 1), between detection threshold and about 1.5-2.0 $\log$ units above threshold, discrimination thresholds for both increments and decrements at first decrease slightly as the reference contrast increases (the "Pedestal Effect" of Leshowitz, Taub \& Raab, 1968). Throughout this paper we shall be concerned only with the data gathered at levels above the pedestal effect range.

For increments, discrimination thresholds increase linearly with increases in the reference value of $\Delta L:$ the value of $\Delta(\Delta L)$ is proportional to $\Delta L$, as one would expect in accordance with a Weber's Law for contrast discrimination. For decrements however, the function is different. As $\Delta L$ increases, $\Delta(\Delta L)$ increases to a maximum value when $\Delta L$ is approximately half the value of $L_{b}$, the background luminance. Beyond this point however, $\Delta(\Delta L)$ decreases with further increases in $\Delta L$.

These generalisations hold true regardless of the luminance of the background. When the data gathered at different background luminances were normalised with respect to detection threshold, all of the functions neatly superimposed. Figure 2, which is an idealised version of Fig. 3 in Whittle (1986), summarises the results described above. Note that although the axes are logarithmic, this is solely in order to compress the range. The linear portion of the functions has a slope very close to unity, which means that it would also be very nearly a straight-line function if plotted on linear axes.

Whittle suggested the following metric of contrast $W$ which linearised all of his data to a good approximation.

$$
W=\Delta L / L_{\min }
$$


(a) Decrements

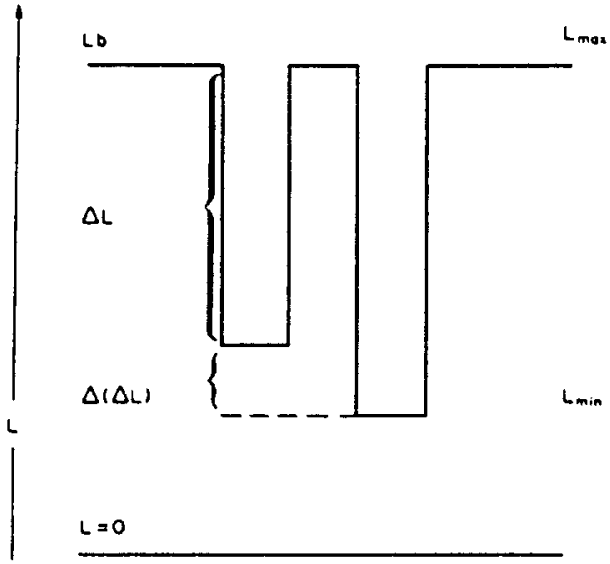

(b) Increments

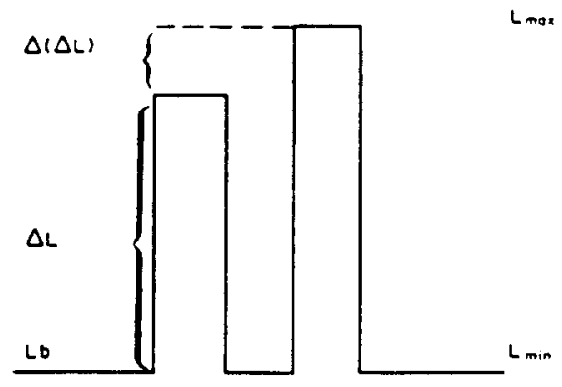

Fig. I. Luminance profiles and terminology for stimuli used by Whittle (1986): (a) decrements:

(b) increments.

where $L_{\min }$ was the smaller of the luminances of the test patch or background. When $\Delta W$ was plotted against $W$ Whittle found he could encompass most of his data by the second-order Weber's Law.

$$
\Delta W / W=k
$$

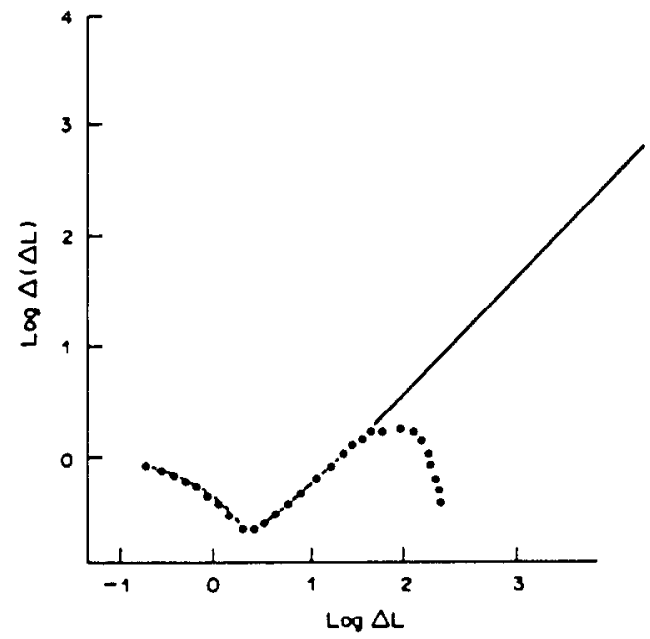

Fig. 2. Idealised plots of the thresholds for contrast discrimination, $\log \Delta(\Delta L)$, as a function of $\log \Delta L$, taken from Fig. 3 of Whittle (1986). Dotted line $=$ decrements; continuous line $=$ increments. Each plot represents data collected at the three levels of background luminance, but normalised with respect to detection threshold.
Figure 3, which is based on Fig. 9 of Whittle (1986), demonstrates this relation. Whittle found that a line of unit slope fitted the data reasonably well.

Given that the $W$ formulation provides such a good fit to the data, it is important to ask



Fig. 3. The same data as in Fig. 2 but plotted in terms of $\Delta W$ against $W$. Note that although the axes are logarithmic, this is simply in order to compress the data. 
whether the metric $W$ has important implications about the precise nature of the properties of the mechanisms underlying contrast discrimination. Whittle $(1986$, p. 1686) puts the issue succinctly: [The equation $\Delta W / W=k$ ] “... suggests a model in which contrast is coded as $W$, but it may be no more than a convenient description...".

Despite the descriptive power of the metric $W$, there are grounds for questioning its plausibility as a basis for modelling. This is simply because while in the case of increments the denominator in the expression for $W$ is the luminance of the background, in the case of decrements it is the luminance of the test patch. The denominator in this and in other Weberlike expressions can be thought of as a scaling factor which reflects the change in gain of the system as a function of stimulus intensity. Why should the gain be set by the background in the case of increments but by the target patch in the case of decrements? At the very least, and even at this level of description, this consideration robs the metric $W$ of what at first sight seemed to be its great unifying power. In what follows we suggest an alternative formulation which avoids this shortcoming.

\section{THE MODEL}

Expressions for contrast of the general type $\Delta L / L_{b}\left(L_{b}=\right.$ background luminance) imply that the response to an increment in luminance added to a steady-state adapting background is a linear function of the magnitude of the luminance increment, with the slope of that function being inversely proportional to the background level. Microelectrode recordings of retinal neurones suggest however that this relationship is not what is observed. In general, the effects of increments and decrements on the peak transient response of single neurones are well described by a compressive non-linearity (such as the Naka-Rushton equation for example), with a semi-saturation constant that is determined by the background level of illumination (Normann \& Werblin, 1974), for mudpuppy cones; Normann \& Perlmann, 1979, for turtle cones; Sakmann and Creutzfeld, 1969. for cat ganglion cells). When test spot luminance is plotted in logarithmic coordinates, the neurones' response is proportional to the logarithm of luminance over the middle part of the range. This is particularly evident in the data provided by Sakmann and Creutzfeld (1969) for cat retinal ganglion cells where the response is proportional to the logarithm of luminance over most of the range of $\Delta L$. The data from Sakmann and Creutzfeldt also show that the effect of the background adapting level is to shift the response curves to the right by an amount proportional to the logarithm of the background luminance. Such evidence has led to the view that a logarithmic transformation of luminance represents a reasonable description of the first transduction stage in vision (Cornsweet, 1970; Legge \& Kersten, 1983). Probably with this assumption in mind the log transform is commonly employed to model the initial transduction stage in models of brightness and lightness constancy (e.g. Land \& McCann, 1971).

Although caution is necessary in extrapolating from single cell recordings to the behaviour of the visual system as a whole, the above considerations suggest a possible alternative description of contrast to that of the $\Delta L / L_{\min }$ formulation. We call it $G$ where:

$$
G=\ln \left(L / L_{b}\right) .
$$

Contrast discrimination thresholds $\Delta G$ are thus given by:

$$
\Delta G=k|G|^{n} ;
$$

with $n$ being the single free parameter whose value is yet to be determined. In what follows we explore the extent to which this alternative formulation may account for Whittle's data.

In general, the response $R$ of an operator to a contrast $C$ can be obtained by a method first proposed by Fechner for establishing the nature of the transducer function of the visual system to luminance (Boring, 1950). Following Fechner, we suppose that a certain fixed amount of change in internal sensation, $\Delta R$, is required for a discrimination threshold $\Delta C$. If one has found empirically that $\Delta C / C^{n}$ is constant, then it is possible to write the general formulation:

$$
\Delta R=k^{\prime} \frac{\Delta C}{C^{n}}
$$

Since $\Delta R$ and $\Delta C$ are small they can be respectively approximated by the limiting values $\mathrm{d} R$ and $\mathrm{d} C$, and $R$ can thus be obtained by integration:

$$
\int \mathrm{d} R=k^{\prime} \int \frac{\mathrm{d} C}{C^{n}}
$$

which gives

$$
\begin{array}{ll}
R=k^{\prime} \frac{C^{1-n}}{1-n}+R^{\prime} & (n \neq 1) \\
R=k^{\prime} \ln C+R^{\prime} & (n=1)
\end{array}
$$


$k^{\prime}$ is a proportionality constant and $R^{\prime}$ the constant of integration ( $R^{\prime}$ is the response when $C=1$ for equation $7 \mathrm{a}$ and $C=0$ for $7 \mathrm{~b}$ ). Expressions of this type have been employed by a number of previous investigators to model the contrast response of the visual system (e.g. Wilson, 1980).

It is thus possible to define the transducer functions of the models of contrast coding which employ the metrics $W$ and $G$ respectively. It is then possible to proceed to adjust any free parameters, such as the value of the exponent $n$ in the latter, to make the transfer function based on $G$ as close as possible to that based on $W$. Moreover, expressions can be derived which describe a number of useful properties of the models, such as the predicted functions relating $\Delta(\Delta L)$ to $\Delta L$ for both increments and decrements.

The transducer functions based on $W$ and $G$, $R_{w}$ and $R_{G}$ respectively, are defined as:

$$
R_{w}=\ln W: \quad R_{G}=\frac{|G|^{1-n}}{1-n} .
$$

The In $W$ function for $R_{w}$ (equation 8a) follows from Whittle's (1986) description of his data in which the $\Delta W / W$ function was fitted by a straight line of approximately unit slope (i.e. an exponent of one). The parameter $R^{\prime}$ in equation $(7 \mathrm{a}, \mathrm{b})$ disappears when the expressions are differentiated (see below) and can therefore be arbitrarily set to zero in equation $(8 \mathrm{a}, \mathrm{b}) ; k^{\prime}$ is an arbitrary scaling factor which we set for simplicity to unity in equation $(8 \mathrm{a}, \mathrm{b})$.

How might we choose that value of the exponent $n$ in the transfer function $R_{G}$ of our model operator which best fits Whittle's data? Refer back to Fig. 2 and the inverse U-shaped curve which describes the function relating $\Delta(\Delta L)$ to $\Delta L$ for decrements. We previously noted that the luminance of the test patch where contrast discriminability was at its worst (the peak in this function) occurred where $L$ equalled approx. $50 \%$ of $L_{b}$, and we refer to the value of $L$ at this point as $L_{m}(m=$ minimum discriminability). In Appendix Al we show on theoretical grounds that the relation $L_{m}=L_{b} / 2$ is exactly what would be expected on the basis of the transducer function $R_{w}=\ln W$. We therefore decided to employ this constraint to set the value of $n$ in the $R_{G}$ equation, and as we show later, the appropriateness of the value of $n$ chosen in this way is confirmed by other means.

In Appendix A2 we show that for our model operator, $L_{m}$ is given by $L_{b} / \mathrm{e}^{n}$, where $e=2.718$.
Thus, if we constrain $L_{m}$ to be $0.5 L_{b}, n$ is calculated to be 0.69 . With the exponent set to that value Figs $4 \mathrm{a}$ and $5 \mathrm{a}$ plot $R_{G}$ as a function of $\Delta L$ for decrements and increments respectively. Also shown is the plot of $R_{w}$ as a function of $\Delta L$, and as can be seen, both transfer functions are very similar in shape.

In Appendix A we show how contrast discrimination thresholds for the $R_{w}$ transducer function are given respectively for increments and decrements by:

$$
\begin{aligned}
& \Delta(\Delta L)_{w}=k L\left(L_{b}-L\right) ; \\
& \Delta(\Delta L)_{w}=k\left(L-L_{b}\right) ;
\end{aligned}
$$

and for the $R_{G}$ transducer function for both increments and decrements by:

$$
\Delta(\Delta L)_{G}=k L\left|\ln \left(L / L_{b}\right)\right|^{n} .
$$

Figures $4 \mathrm{~b}$ and $5 \mathrm{~b}$ show the functions $\Delta(\Delta L)_{G}$ and $\Delta(\Delta L)_{w}$ for decrements (4b) and increments (5b) respectively, and as expected the functions based on $R_{G}$ and $R_{w}$ are very similar.

It is now possible to examine more closely the extent to which our model fits Whittle's data. For this stage of the argument it is necessary to be quite clear about the distinction between $W$ as a description of the contrast of individual stimuli, and the expressions $R_{w}=\ln W$ or $\Delta W / W=k$ which refer to the properties (respectively the transducer function and the discrimination function) of a theoretical model of contrast coding in the visual system. Whilst we are arguing against the expression as a basis for model, the ratio $W$ (like any expression that describes the relationship between the luminances of the test patch and its background) is a perfectly acceptable metric for stimulus contrast.

Thus we can take some value of $W$ for a given background luminance: the luminance of the test patch is then determinate [since $\left.W=\left(L_{\max }-L_{\min }\right) / L_{\min }\right]$. From that pair of luminances we can calculate, from a model, what the expected threshold contrast difference will be between this original stimulus and another on the same background. The contrast of this second stimulus can then be expressed in terms of $W$, and the difference $\Delta W$ can be computed. It is thus possible to plot the predicted value of $\Delta W$ against $W$ according to any model of one's choosing.

Refer now to Fig. 3, which describes the plot of the empirical values of $\Delta W$ against $W$. This is the plot which is fitted by the straight line 

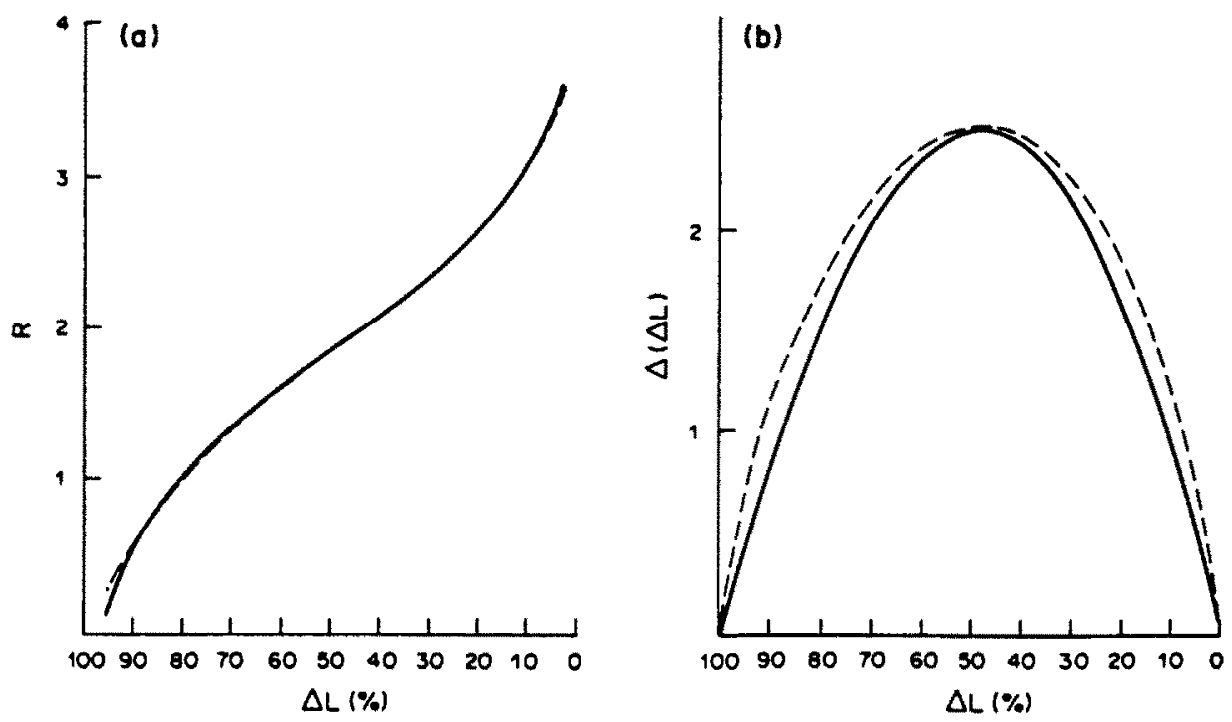

Fig. 4. Theoretical functions for models of contrast coding of decrements based on the metrics $W$ and $G$. Continuous lines $=$ functions based on $W$; dashed lines $=$ functions based on $G$ with an exponent $n=0.69$ (see text for details). (a) Transducer function, (b) $\Delta(\Delta L)$ functions. The abscissae are plotted in linear units of $\Delta L$ as a percentage of $L_{b}$. The ordinate is also in linear units. In (a) the intercepts and scaling constants of each curve have been arbitrarily chosen to bring the functions into close correspondence in order that their shapes may be readily compared. In (b) the plots have been scaled to bring the ordinate values at $\Delta L=50 \%$ into alignment.

function $\Delta W / W=k$ to a first approximation, and which therefore suggests the possibility of a model of contrast coding whose transfer function is given by $R_{w}$. How closely are the empirical values of $\Delta W$ matched by those predicted by our model whose transfer function is $R_{G}$ ? To answer this question we have replotted a representative set of data points taken from Fig. 9 of Whittle (1986). The points were from subject $P W$ and were the data set obtained with a background luminance of $2.35 \mathrm{log}$ td for the

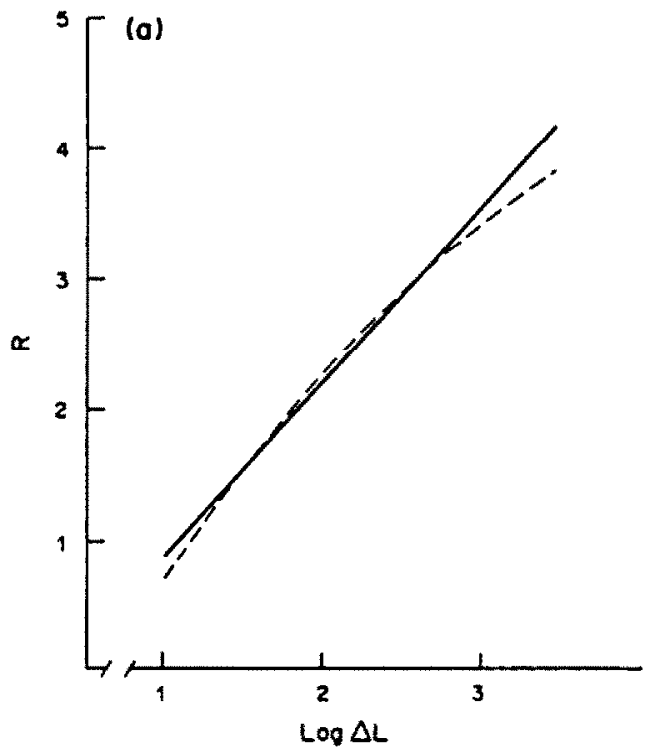

decrements and $3.35 \log$ td for the increments (there were insufficient data points in the increment data at the background luminance of 2.35 td). The decrement values are shown in Fig. 6a, the increment values in Fig. 6 b.

First refer to the continuous lines in Fig. 6a and $b$. These represent the straight line fits of $\Delta W / W=k$, with $k$ set to 0.12 , the value Whittle found to give the best fit to the data as a whole. Notice that for the decrements (Fig. 6a), the data points diverge upwards away from the

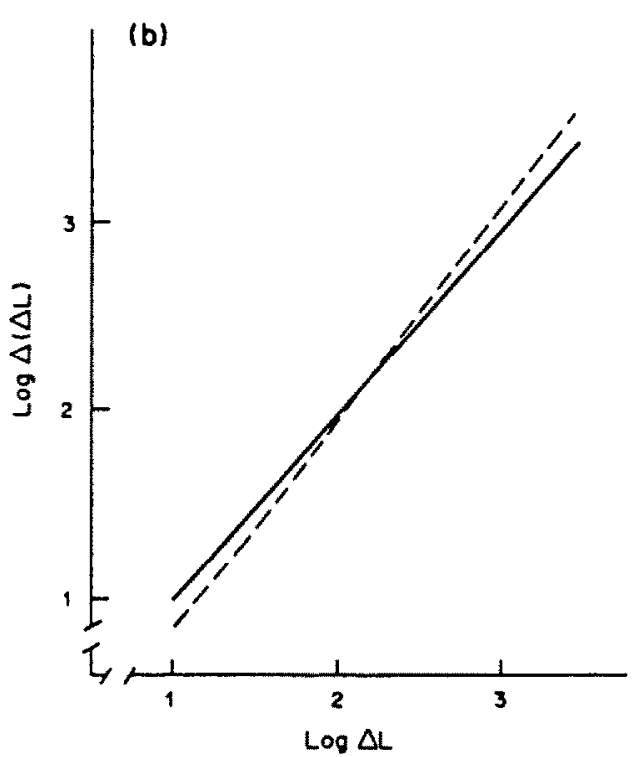

Fig. 5. Theoretical functions as in Fig. 4 for increments. 

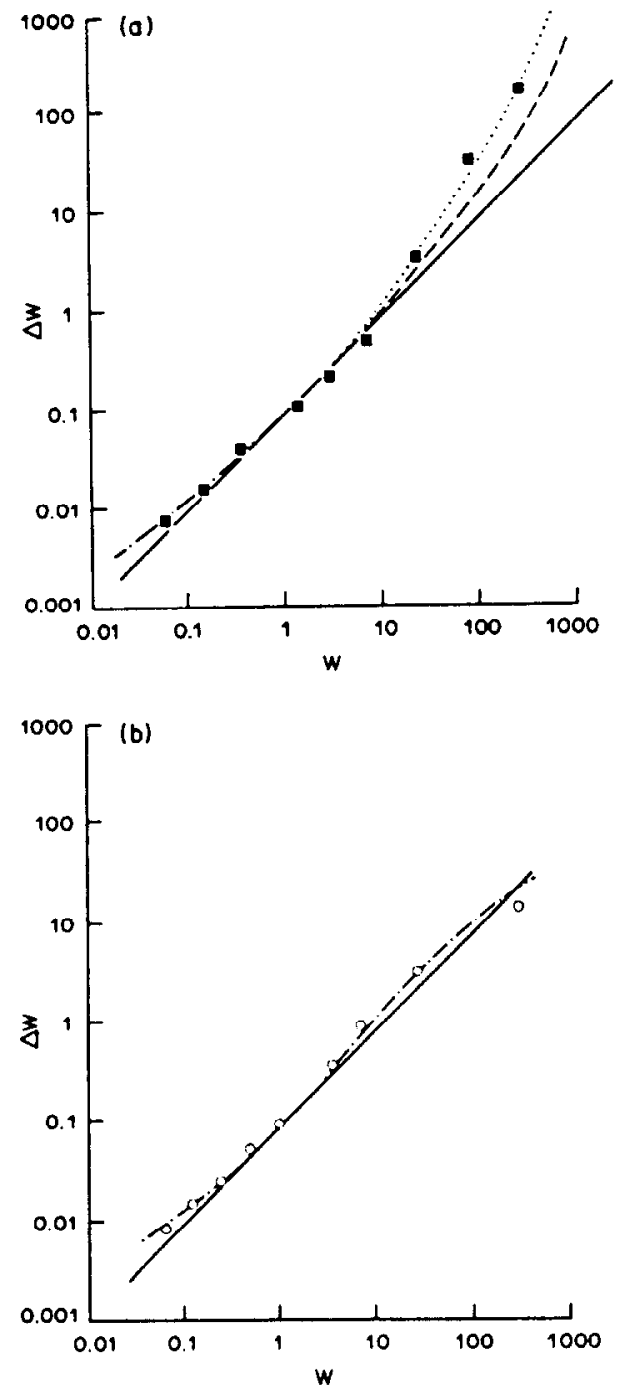

Fig. 6. Predicted functions of $\Delta W$ as a function of $W$ according to two models. The data points represent actual empirical values taken from Fig. 9 of Whittle (1986). (a) Decrements: solid squares are data points from subject PW at a background of $2.35 \mathrm{log}$ td. (b) Increments: open circles are data points from PW at a background of $3.35 \log$ td. Continuous lines represent the function $\Delta W / W=0.12$. Dashed lines are the $\Delta W_{G}$ function derived from the Transducer function $R_{G}$ (see text for details) with an exponent of $n=0.69$ and scaling constant $k=0.06$. Dotted lines represent the $\Delta W_{G}$ function in the modified version of $R_{G}$ which includes the parameter $L_{n}$ set to 2.0 td (see text for details).

continuous line at large values of $\Delta L$. This divergence is found for all the decrement data that Whittle collected (see his Fig. 9), and represents a modest though important limitation on the predictive power of the $R_{w}=\ln W$ model.

We define the value of $\Delta W$ that is predicted by our model as $\Delta W_{G}$, and in Appendix $C$ we show that:

$$
\begin{aligned}
& \Delta W_{G}=\frac{L_{b} \Delta(\Delta L)_{G}}{L\left[L-\Delta(\Delta L)_{G}\right]} ; \\
& \Delta W_{G}=\frac{\Delta(\Delta L)_{G}}{L_{b}}
\end{aligned}
$$

for decrements and increments respectively. with $\Delta(\Delta L)_{G}$ as previously defined in equation (9). These functions are plotted as the dashed lines in Fig. 6a and b respectively, with the constant of proportionality $k$ in equation (9) set to 0.6 (the value that gave the best fit in the final stage of modelling described below).

For the increment data the fit of the $R_{G}$ model (dashed line) is at least as good as the predictions based upon $R_{w}$ (continuous line); moreover the $R_{G}$ predictions for the decrement data (dashed line), being slightly bow-shaped, fit those data even more closely that those from $R_{w}$ (continuous line). In particular, this initial version of $R_{G}$ already begins to reflect the upward divergence of the values of $\Delta W$ at high contrasts.

These high contrast values of $\Delta W$ in the decrement data in Fig. 6a still appear to be even higher than the simple version of our model predicts and we now consider why this might be so. Inspection of Whittle's (1986) Fig. 9 reveals two features. First, there is a considerably greater degree of dispersion in these high contrast data points than elsewhere on the function, and second, this dispersion is orderly. In each case the data gathered at lower background luminances give higher discrimination thresholds.

It is known that although over much of its range the response of the visual system is a linear function of the logarithm of luminance (Weber's Law), at low luminances the response function flattens out (Whittle \& Challands, 1969). In equation (1), which contains a description of the initial non-linear transformation of luminance by our model operator, the input luminance is simply log transformed: we have assumed up to this point that Weber's Law holds across the whole range of luminance. The departure from Weber's law at low luminance is partly a consequence of noise in the neural transduction process but principally a consequence of the particular way in which the gain, or adaptational level, of the retina changes with the luminance level (Shapley \& Enroth-Cugell, 1984). Gain is defined as the rate of growth of response to luminance and Shapley and EnrothCugell (1984) have suggested that a description 
of the gain for retinal ganglion cells may be given by:

$$
\text { Gain }=\frac{\mathrm{d} R}{\mathrm{~d} L}=\frac{K}{1+L / L_{\mathrm{h}}}
$$

where $K$ is the gain for the completely dark adapted state and $L_{h}$ the luminance at which that gain is halved. We employ equation (12) as an engineering approximation to the change of gain with luminance that occurs in the retina as a whole, as we have done previously (Kingdom \& Moulden, 1989). The consequence of equation (12) for the transducer function which describes the growth of response of the visual system to luminance is obtained by integration of equation (12) which gives:

$$
R=K L_{h} \ln \left(1+L / L_{h}\right)+C .
$$

If one substitutes equation (13) for both $\ln L$ and $\ln L_{b}$ (with $L_{b}=L$ in the latter) in equation (8a). then after simplification the transducer function of the model is modified to the form:

$$
R_{c}=\frac{K L_{h}}{1-n}\left|\ln \left\{\frac{L+L_{h}}{L_{b}+L_{h}}\right\}\right|^{l-n} .
$$

Equation (14) may be simplified even further by setting the (arbitrary) constant of proportionality $K L_{h}$ equal to unity. There are now two free parameters, $n$ and $L_{h}$. We found by iterative search that the combination of $n$ and $L_{n}$ which best fitted the data points in Fig. 6a was $n=0.69$ and $L_{h}=2.0 \mathrm{td}$. The value of $n=0.69$ arrived at in this way was identical to that arrived at by the alternative method described earlier.

The resulting predictions for $\Delta W_{G}$ (see Appendix $B$ for mathematical details) are shown as the dotted lines in Fig. 6. For the increment data (Fig. 6b), the addition of the constant $L_{h}$ makes no difference to the predictions, as would be expected.

On the other hand for the decrement data (Fig. 6a), the addition of $L_{h}$ produces an upwards shift in the function at high contrasts, and the resulting fit to the data is now excellent. Moreover, the modified version of $R_{G}$ correctly predicts the other feature of Whittle's data that we noted above, namely that at high decrement contrasts there is an ordered increase in the values of $\Delta W$ with lower and lower background luminance. This occurs, according to our model, because the proportional contribution that $L_{h}$ makes to the $R_{G}$ function increases as $L_{b}$ and $L$ decreases, producing a greater and greater upward shift in the $\Delta W_{G}$ function at high contrast.

\section{SUMLMARY AND CONCLUSION}

We have shown how a model based upon the logarithmic transform of stimulus luminance can provide a very good fit to the data of Whittle (1986). In its simplest version, the model has one free parameter, the exponent in the power function that describes contrast discrimination thresholds. When this free parameter was suitably adjusted the fit to Whittle's data was as good as with the metric $W$ (also fitted with one free parameter). With the inclusion of a second free parameter, which took into account a nonlinearity in the gain-luminance relationship at low luminance levels, the fit was even better.

Acknowledgements - This work was supported by a Science and Engineering Research Council (SERC) U.K. grant under the auspices of the Special Initiative on Image Interpretation (GR/D 89165).

\section{REFERENCES}

Boring. E. G. (1950). The history of experimental psychology (p. 288). New York: Appleton, Century Crofts.

Cornsweet. T. (1970). Visual perception. New York: Academic Press.

Kingdom, F. \& Moulden. B. (1989). Modelling visual detection: Luminance response non-linearity and internal noisc. Quarterly. Journal of Experimental Psycholegy, 4IA, 675-696.

Land, E. H. \& McCann, J. J. (1971). Lightness and retinex theory. Journal of the Optical Society of America, 6l, I-11.

Legge, G. E. \& Kersten, D. (1983). Light and dark bars: Contrast discrimination. Vision Research, 23, 473-483.

Leshowitz, B., Taub, H. B. \& Raab, D. H. (1968). Visual detection of signals in the presence of continuous and pulsed backgrounds. Perception and Psychophysics, 4, 207-213.

Normann, R. A. \& Perlman, I. (1979). The effects of background illumination on the photoreceptors of red and green cones. Journal of Physiology, London, 286, 491-507.

Normann. R. A. \& Werblin. F. S. (1974). Control of retinal sensitivity-I. Light and dark adaptation of vertibrate rods and cones. Journal of General Physiology, 63, 37-61.

Sakmann, B. \& Creutzfeldt, O. D. (1969). Scotopic and mesopic light adaptation in the cat's retina. Pflugers Archiv, 313, 168-185.

Shapley, R. \& Enroth-Cugell, C. (1984). Visual adaptation and retinal gain controls. In Osborne. N. G. \& Chader, G. J. (Eds.), Progress in retinal research (Vol. 3, pp. 263-343). Oxford: Pergamon Press.

Whittle. P. (1986). Increments and decrements: Luminance discrimination. Vision Research, 26, 1677-1691.

Whittle, P. \& Challands, P. D. C. (1969). The effect of background luminance on the brightness of flashes. Vision Research, 9, 1095-1110. 
Whittle, P. \& Swanston, M. T. (1974). Luminance discrimination of separated fiashes: The effect of background luminance and the shapes of tvi curves. Vision Research. 14. $713-719$.

Wilson. H. R. (1980). A transducer function for threshold and suprathreshold vision. Biological Cybernetics, 38, 171-178.

\section{APPENDIX A}

\section{Theoretical Properties of the $R_{w}$ Transfer Function}

The sensitivity of a model operator to small changes in the luminance $L$ of the test patch (i.e. to small changes in contrast) is approximated by the first derivative with respect to $L$ of the transfer function of the operator. The $\Delta(\Delta L)$ function is then approximated by the reciprocal of this first derivative. scaled by a constant $k$ which is determined empirically.

The sensitivity to small changes in $L$ for decrements is given by:

$$
\frac{\mathrm{d} R_{w}}{\mathrm{~d} L}=\frac{\mathrm{d}}{\mathrm{d} L} \frac{\ln L_{b}-L}{L} ;=\frac{-L_{b}}{L\left(L_{b}-L\right)} ;
$$

and thus the (absolute) values of $\Delta(\Delta L)_{w}$ are given by

$$
\Delta(\Delta L)_{\omega}=\frac{k L\left(L_{\mathrm{A}}-L\right)}{L_{\mathrm{A}}} .
$$

Equation (A2) is an inverse $U$ shaped function when $\Delta(\Delta L)_{w}$ is plotted against $L$ for a given value of $L_{n}$. To obtain that value of $L$ where the function peaks, $L_{m}$, we set the derivative with respect to $L$ to zero and solve for $L$. Thus:

$$
\frac{\mathrm{d}}{\mathrm{d} L} \Delta(\Delta L)_{\omega}=\frac{k\left(L_{\mathrm{A}}-2 L\right)}{L_{\mathrm{h}}}
$$

selting equation (A3) equal to zero, substituting $L_{m}$ for $L$ and solving for $L_{m}$ gives:

$$
L_{m}=L_{h} / 2 \text {. }
$$

Equation (A4) implies that the peak value of $\Delta(\Delta L)$ will always occur when $L$ (or $\Delta L$ ) is $50 \%$ of the value of $L_{b}$.

For increments the sensitivity to small changes in $L$ is approximated by:

$$
\frac{\mathrm{d} R_{\mathrm{w}}}{\mathrm{d} L}=\frac{\mathrm{d}}{\mathrm{d} L} \ln \left\{\frac{L-L_{b}}{L_{\mathrm{b}}}\right\}=\frac{1}{L-L_{b}} .
$$

Thus, the $\Delta(\Delta L)_{w}$, function for increments is given by:

$$
\Delta(\Delta L)_{w}=k\left(L-L_{b}\right)
$$

\section{APPENDIX B}

\section{Theoretical Properties of the $R_{G}$ Transfer Function}

From the argument given in Appendix $A$, the sensitivity to small changes in $L$ for the $R_{G}$ transfer function is approximated by:

$$
\frac{\mathrm{d} R_{G}}{\mathrm{~d} L}=\frac{1}{1-n} \frac{\mathrm{d}}{\mathrm{d} L}\left|\ln \left(L / L_{b}\right)\right|^{1-n}=\left|\ln \left(L / L_{b}\right)\right|^{-n} \frac{1}{L} .
$$

And thus the $\Delta(\Delta L)_{G}$ function for both decrements and increments is given by:

$$
\Delta(\Delta L)_{G}=k L\left|\ln \left(L / L_{b}\right)\right|^{n} .
$$

To obtain the peak value of $\Delta(\Delta L)_{G}$ we follow the method described in Appendix A:

$$
\begin{aligned}
\frac{\mathrm{d}}{\mathrm{d} L} \Delta(\Delta L)_{G} & =k \frac{\mathrm{d}}{\mathrm{d} L} L\left|\ln \left(L / L_{b}\right)\right|^{n} \\
& =k L n\left|\ln \left(L / L_{b}\right)\right|^{n-1} 1 / L+k\left|\ln \left(L / L_{b}\right)\right|^{n} .
\end{aligned}
$$

Setting this result equal to zero, simplifying and setting $L_{m}$ equal to $L$ gives:

$$
L_{m}=L_{b} / e^{n} .
$$

The $\Delta(\Delta L)_{G}$ function for the modified version of the $R_{G}$ transfer function containing the parameter $L_{n}$ is obtained in the same way as described above.

$$
\begin{aligned}
\frac{\mathrm{d} R_{C}}{\mathrm{~d} L} & =\frac{\mathrm{d}}{\mathrm{d} L} \frac{1}{1-n}\left|\ln \left\{\frac{L+L_{h}}{L_{b}+L_{h}}\right\}\right|^{1-n} \\
& =\frac{1}{L+L_{h}}\left|\ln \left\{\frac{L+L_{h}}{L_{b}+L_{h}}\right\}\right|^{-n} .
\end{aligned}
$$

Taking the reciprocal and incorporationg the constant $k$ gives:

$$
\Delta(\Delta L)_{i}=k\left(L+L_{n}\right)\left|\ln \left\{\frac{L+L_{h}}{L_{h}+L_{h}}\right\}\right|^{n} .
$$

\section{APPENDIX C}

\section{Estimation of $\Delta W_{G}$}

To obtain the predicted values of $\Delta W$ from the $R_{C}$ transfer function we define $\Delta W$ in terms of $\Delta(\Delta L)$, and simply substitute $\Delta W_{G}$ for $\Delta W$ and $\Delta(\Delta L)_{G}$ (equation $\mathrm{B} 2$ ) for $\Delta(\Delta L)$.

For decrements

$$
\Delta W=\frac{L_{b}-[L-\Delta(\Delta L)]}{L-\Delta(\Delta L)}-\frac{\left(L_{b}-L\right)}{L} .
$$

Substituting $\Delta W_{G}$ for $\Delta W$ and $\Delta(\Delta L)_{G}$ for $\Delta(\Delta L)$ gives, after simplification:

$$
\Delta W_{G}=\frac{L_{b} \Delta(\Delta L)}{\Delta(\Delta L)[L-\Delta(\Delta L)]} .
$$

For increments:

$$
\Delta W=\frac{[L+\Delta(\Delta L)]-L_{\mathrm{b}}}{L_{\mathrm{b}}}-\frac{L-L_{\mathrm{b}}}{L_{\mathrm{b}}} ;
$$

and by substitution as with decrements:

$$
\Delta W_{G}=\frac{\Delta(\Delta L)_{G}}{L_{b}} .
$$

